\title{
Lateral Forces on a Sphere
}

\author{
J.P. Matas ${ }^{1}$, J.F. Morris ${ }^{2}$ and E. Guazzelli ${ }^{1}$ \\ 1 IUSTI - CNRS UMR 6595, Polytech'Marseille, Technopôle de Château-Gombert, 13453 Marseille Cedex 13 - France \\ 2 Halliburton, 2600 S. 2nd St., Duncan, OK 73536 - United States \\ e-mail: jean-philippe.matas@polytech.univ.mrs.fr - elisabeth.guazzelli@polytech.univ.mrs.fr
}

Résumé - Migration latérale sous écoulement - Le transport de particules dans une conduite, et de façon plus générale dans un écoulement cisaillé, a longtemps été modélisé en supposant la concentration uniforme. Pourtant, dès 1836, Poiseuille observe des distributions radiales non homogènes dans ses études sur le comportement des globules rouges dans le sang.

L'observation expérimentale d'une répartition inhomogène des particules dans plusieurs écoulements cisaillés a cependant conduit à identifier différents types de mécanismes de migration. Avant d'aborder les mécanismes de migration collectifs, essentiellement actifs à bas nombre de Reynolds, nous décrivons les mécanismes de migration «à une particule », généralement observés en présence d'inertie. Une courte partie sur les migrations latérales pour des fluides viscoélastiques sera également abordée. La dernière partie aborde le rôle de la sédimentation sur les effets latéraux.

\begin{abstract}
Lateral Forces on a Sphere - The observation of inhomogeneous radial distributions of particles in tube flow dates from the work of Poiseuille (1836) who was mainly concerned by the flow of blood and the behavior of the red and white corpuscles it carries. These results were then generalized to non-biological flows and experiments on pipe flow of suspensions also indicated that significant deviations from ideal Poiseuille flow could occur in the presence of particles.

We will consider systems where the fluid flow in the absence of particles is unidirectional. We will first present how fluid-particle interactions can induce lateral migration in the case of a single rigid particle in a shear flow, as a function of the Reynolds number. While the focus is upon inertial migration, a brief discussion of lateral migration in polymeric and viscoelastic fluids, where the nonlinearity results from the non-Newtonian behavior of the suspending fluid, will be presented at the conclusion of this Section. The role of interparticle interactions in a sheared fluid will be considered in the third section in the case of Stokes flow. The last section will briefly present how sedimentation can affect lateral motion.
\end{abstract}




\section{EARLY EXPERIMENTAL OBSERVATIONS}

The observation of inhomogeneous radial distributions of particles in tube flow dates from the work of Poiseuille (1836) who was mainly concerned by the flow of blood and the behavior of the red and white corpuscles it carries. Poiseuille observed that there was a region devoid of particles close to the walls of the tube, an observation later confirmed by Fåhraeus and Lindquist (1931). These results were generalized to nonbiological flows by Scott-Blair (1930) and later Maude and Whitmore (1956).

Experiments on the rheology of blood and more generally on pipe flow of suspensions also indicated that significant deviations from ideal Poiseuille flow could occur in the presence of particles. It was, for instance, observed that suspensions flowed "relatively more readily" in narrow than wider capillaries, an effect mentioned for the first time in 1919 by Bingham and Green for the flow of paints. This reduction in viscosity observed when a suspension was flowed through a thin capillary, termed the sigma-effect by Scott-Blair (1930), was believed to be related to a lateral migration of the suspended particles towards regions of low shear, tending again to confirm the existence of lateral motion in unidirectional flows. The review paper of ScottBlair (1958) gives a review of most of the anomalous viscosity behaviors observed up to its date of publication. In 1962, Segré and Silberberg provided an extensive and quantitative study of a radial migration phenomenon. Surprisingly, they gave evidence of a lateral migration directed towards the wall of a tube, i.e. opposed to most of the previous experimental observations. Their work triggered a series of experimental and theoretical studies, but before looking at them we need to establish more precisely the framework of this problem.

We will consider systems where the fluid flow in the absence of particles is unidirectional. The lateral migration of a spherical neutrally-buoyant particle is a "nonlinear" phenomenon, in the sense that any lateral force can only depend nonlinearly upon the unperturbed fluid velocity. To illustrate that, the typical example is that of a rigid sphere suspended in Poiseuille flow of a Newtonian fluid at vanishing Reynolds number, i.e. for linear conditions. If under axial flow in a given direction the particle underwent lateral migration towards the wall, it would be expected to migrate towards the centreline under flow reversal (based upon the reversibility arising from linearity of the Stokes equations). However due to the symmetry of the problem the two situations are incompatible, and migration is therefore disallowed at zero Reynolds number. The nonlinearity necessary to achieve migration can potentially arise from the presence of inertia (finite Reynolds number flows), or from any non-Newtonian effect. In particular, the rheology of finite volume fraction suspensions is generally nonNewtonian owing to a range of factors including irreversible particle interactions. Hence, lateral migration phenomena of suspensions in Newtonian fluids are expected to depend upon the particle volume fraction and the Reynolds number. Deformation of the suspended bodies can also introduce rate dependence and therefore nonlinearity, but we will restrict our attention to the migration of rigid particles. Lateral motion of deformable particles, relevant in the case of drops or red cells in blood flow, is addressed in Leal (1980), Legendre and Magnaudet (1998) and the references therein. We will first present how fluid-particle interactions can induce lateral migration in the case of a single rigid particle in a shear flow, as a function of the Reynolds number. While the focus is upon inertial migration, a brief discussion of lateral migration in polymeric and viscoelastic fluids, where the nonlinearity results from the non-Newtonian behavior of the suspending fluid, will be presented at the conclusion of this section. The role of interparticle interactions in a sheared fluid will be considered in the third section in the case of Stokes flow. The last section will briefly present how sedimentation can affect lateral motion.

\section{SINGLE PARTICLE MIGRATION}

\subsection{Experimental Observations}

Single particle experimental situations present a twofold interest. They serve as a useful validation for lateral migration analytical models, which generally cannot deal with many-particle effects, but they also help understand migration patterns still present at finite but low concentrations. Bretherton (1962) pointed out the absence of lateral migration for a rigid isotropic body in the zero Reynolds number unidirectional flow of a Newtonian fluid, and thus the importance of finite Reynolds number effects in single particle issues.

The behavior of a neutrally buoyant rigid sphere in pipe flow was studied experimentally by Segré and Silberberg (1962). They observed that a rigid sphere in a cylindrical Poiseuille flow (pipe of radius $R$ ) migrated to an equilibrium position located at $r=0.62 R$ in conditions of small but finite Reynolds number (Fig. 1). The effect was called the tubular pinch effect, because of the tube-like shape of the annular region to which particles migrate.

Their experiment prompted a strong interest in the suspension community, mainly because the theoretical evidence to support lateral migration at the time (Saffman, 1956) could only predict lateral forces directed towards the centre of the pipe.

These results were confirmed by several following experimental studies. The case of non neutrally buoyant particles in vertical Poiseuille flow was also studied experimentally by several authors (Oliver, 1962; Repetti and Leonard, 1964; Jeffrey and Pearson, 1965; Karnis et al., 


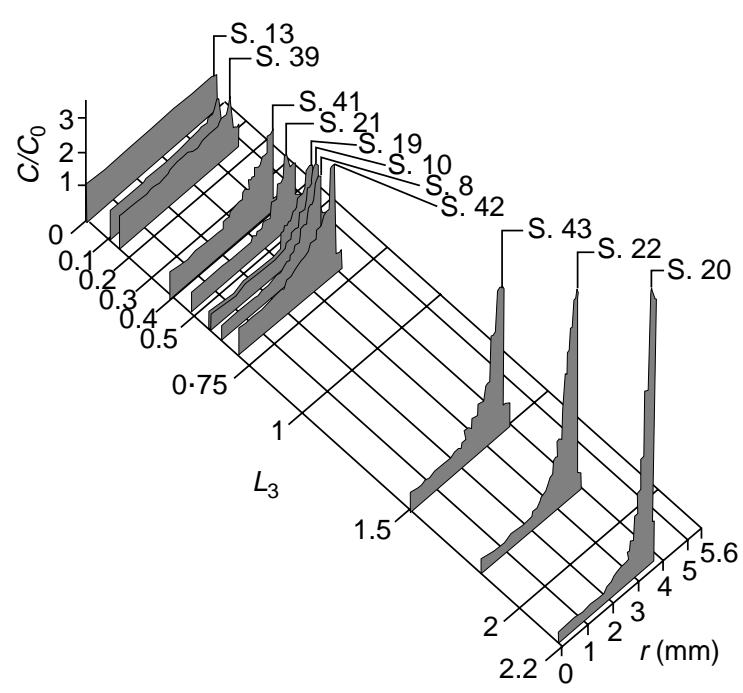

Figure 1

Experimental results of Segré and Silberberg (1962), showing how radial migration develops in a pipe from a uniform concentration at the entrance. The pipe radius is $r=5.6 \mathrm{~mm}$.

1966; Tachibana, 1973; Aoki et al., 1979). They found that the equilibrium position for nonneutrally buoyant particles may be moved either towards the axis or towards the wall depending upon the relative densities of the fluid and the particle. In vertical ascending Poiseuille flow for instance, particles heavier than the fluid (thus going slower) can be observed to migrate towards the centre of the pipe, whereas a particle which is less dense than the fluid (thus going faster) will migrate towards the wall. Some of these studies also investigated the behavior of rigid particles in other sheared flows. In plane Couette flow for instance, neutrally buoyant particles are observed to migrate towards the median plane. If the particles have a different density, this symmetry is broken: in a frame where one of the plates is stationary particles will migrate towards the moving plate if they lag the flow, and conversely towards the fixed plate if they lead the flow. Mechanisms for these migration phenomena were suggested first by Saffman (1965), then specified to the case of bounded and quadratic flows by Ho and Leal (1974) and Vasseur and Cox (1976). In the next Section we present an outline of their respective approaches as well as a suggestion of the physical mechanisms which come into play.

\subsection{Mechanisms of Single Particle Migration}

Segré and Silberberg's experiment aroused a strong interest in the fluid mechanics community, since it gave the first quantitative experimental evidence of lateral migration of a sphere in the unidirectional flow of a Newtonian fluid. Bretherton (1962) had shown that in Stokes flow no lateral migration could occur for rigid spherical particles in a unidirectional flow. Hence, in order to predict Segré and Silberberg's experimental result finite Reynolds number studies were undertaken to include inertial effects. The first analytical studies addressed the problem of lift forces on a particle in a slightly inertial flow, and considered the simplest case of unbounded flows. One of the first analyses of this type was performed by Rubinow and Keller (1961). They calculated, via a matched asymptotic expansion method, the lift force on a rigid sphere translating in a quiescent fluid with a rotation perpendicular to the translation, in the limit of small Reynolds numbers. They found that in such conditions a sphere experiences a force given by $F_{R K}=\pi a^{3} \rho \Omega \times U$, where $a$ is the radius of the sphere, $\rho$ the fluid density, $\Omega$ the rotation vector and $U$ the velocity of the fluid. Though this particular expression of the lateral force on a sphere is only valid for low Reynolds numbers, this type of force due to the rotation of a body in a uniform flow is present in more strongly inertial flows, since its physical origin is contained in Bernoulli's theorem: the rotation creates a reduction in pressure on the side where rotation tends to increase the velocity of the fluid (Fig. 2). It accounts for the curving of the trajectory of a spinning ball. Rubinow and Keller's force does not depend on the viscosity, and this is consistent with the perfect fluid mechanism suggested by Bernoulli's theorem.

In the case of Poiseuille flow, force-free neutrally buoyant particles are known to lag behind the fluid (even at vanishing Reynolds number), as the result of the finite size influence proportional to $a^{2} \nabla^{2} U$ in the Faxén law (Kim and Karrila, 1991). This combined with the rotation of the particle induced by the shear yields a lateral force of the form mentioned above, but this force is in this case directed towards the axis, and thus cannot explain Segré and Silberberg's observations. This indicates that the Rubinow-Keller force, though present, is not the dominant contribution to the lateral force exerted on a neutrally buoyant particle.

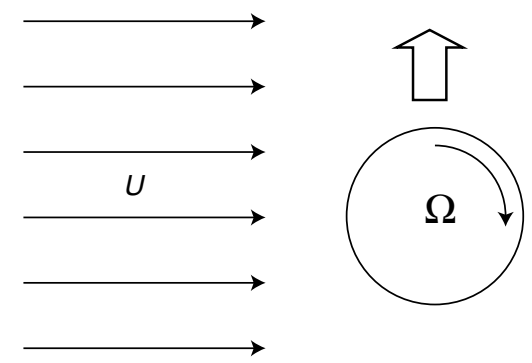

Figure 2

Force exerted on a rigid sphere in the uniform flow of a perfect fluid (Magnus effect): $U$ is the velocity of the fluid, $\Omega$ the rotation vector. The force is directed towards the larger velocity side (in a frame moving with the particle). 
Saffman (1965) extended Rubinow and Keller's calculations to the case of a simple unbounded shear flow. The lift force is calculated via the matched asymptotic expansion method. Saffman found that to largest order in the reciprocal of the viscosity the particle experiences a lift force induced by the shear. This force can be seen as arising from the interaction of the Stokeslet velocity field created by the particle with the velocity gradient. The magnitude of this force is $F_{S}=K a^{2} \gamma^{-1 / 2}(\mu \rho)^{1 / 2} V$, where $K$ is a numerical constant $(K \sim 6.46), \gamma^{-1}$ is the rate of shear, $\mu$ is the dynamic viscosity of the Newtonian fluid and $V$, the relative velocity of particle and fluid measured on the streamline through the centre of the particle. This lift force, unlike the RubinowKeller force, is independent of particle rotation.

The direction of the force can be found by noting that the sphere will migrate towards the side where the fluid velocity (relative to the particle) is the largest (Fig. 3). A comparison between $F_{R K}$ and $F_{S}$ can be made in the simplest case $\Omega=\gamma^{1 / 2}$ (freely rotating particle), and shows that the condition $F_{R K}<F_{S}$ is equivalent to $\mathrm{Re}^{1 / 2}<K$, i.e. $\operatorname{Re}<6600$, and thus valid in the present case of small Reynolds numbers. Consequently, Saffman's force, due to the interaction of slip velocity and shear, will generally be at least one order of magnitude larger than Rubinow-Keller's force due to the interaction of slip velocity and rotation of the particle. In the case of a neutrally buoyant particle in Poiseuille flow, the particle is force-free and cannot generate a Stokeslet in the base flow, so there is no Saffman force (the lag of the particle relative to the flow generates a Stokeslet, but it is balanced by an opposite Stokeslet emanating from the curvature of the base flow). Saffman's force is however relevant in the case of non neutrally buoyant particles, since those create a Stokeslet in the flow. The evolution of lateral migration with sedimentation observed by Jeffrey and Pearson (1965) in Poiseuille flow is consistent with the influence of this lateral force when the particle is heavier of lighter than the suspending fluid.

Although the previous results helped understand some of the physics of the Segré-Silberberg effect in the case of nonneutrally buoyant particles, they cannot explain SegréSilberberg's observations for neutrally-buoyant particles. By a regular perturbation method including the influence of the walls and the quadratic nature of the unperturbed fluid velocity, Ho and Leal (1974) and later Vasseur and Cox (1976) eventually predicted most of the experimental observations for neutrally or nonneutrally buoyant particles in linear or quadratic flow. Both studies show that in conditions of low Reynolds number, neutrally buoyant particles in a simple shear Couette flow will migrate towards the centre plane because of the influence of the walls (agreeing with experimental observations by Halow and Wills 1970). In quadratic Poiseuille flow there will be in general a competition between an inwards force and an outwards force, leading to an equilibrium position at about $60 \%$ from the centre plane to the walls at small Re (Segré and Silberberg experimental result).

To understand the physical mechanisms controlling the lateral migration of a rigid sphere in a shear flow, we can follow Feng et al. (1994) and distinguish in the global lift force three different types of contributions:

- The interaction of a Stokeslet arising from any nonhydrodynamical force on the particle with the simple shear component of the fluid velocity will generate a Saffman contribution to the lift force. This type of force is dominant in the case of nonneutrally buoyant particles in a vertical flow: for a vertical ascending plane Couette flow for instance particles will migrate towards the mobile plate if they are heavier than the fluid and hence lag the flow. If on the contrary they are lighter than the fluid and lead the flow, migration will be towards the still plate (Fig. 4).
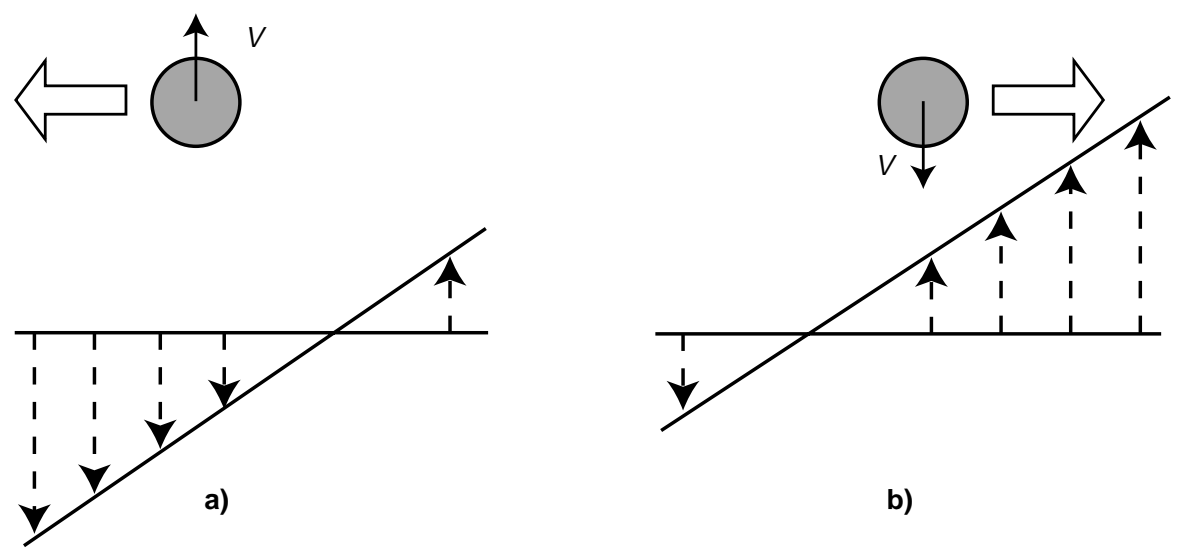

Figure 3

Force exerted on a rigid sphere in a simple shear flow (Saffman, 1965): $V$ is the velocity of the sphere, and the fluid velocity is zero for the streamline going through the sphere centre. The dotted fluid velocity is relative to the sphere velocity; a) $V>0$, the sphere moves faster than the fluid; b) $V<0$, it is going slower than the fluid. 


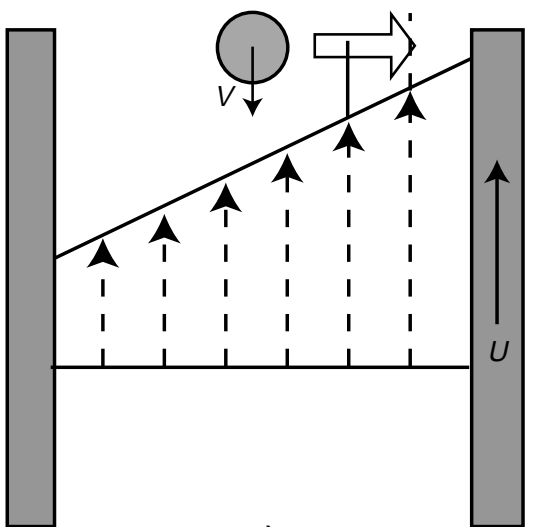

a)

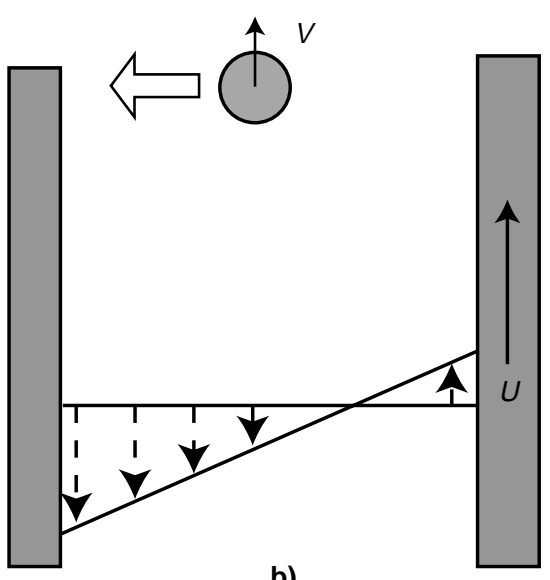

b)

Figure 4

Force exerted on a nonneutrally buoyant rigid sphere in a vertical plane Couette flow: $V$ is the absolute velocity of the sphere, $U$ the velocity of the mobile plate. The dotted fluid velocity is relative to the sphere velocity; a) the particle is denser than the fluid; b) the particle is less dense than the fluid.

- Walls tend to push away the particles. This effect makes a particle which sediments in a quiescent fluid or a neutrally buoyant particle in a plane Couette flow migrate to a position equidistant from both walls. For a particle moving parallel to a wall, the pressure field around the sphere will be greater on the wall side, and will consequently make the mid-plane an equilibrium position. When the particle is nonneutrally buoyant, there is a competition between the wall forces and Saffman's force, and the balance between them will determine the equilibrium position.

- There is a third source of migration, important in the case of quadratic flows and needed to explain Segré and Silberberg's results. This mechanism is related to the curvature of the velocity profile in such flows (Ho and Leal, 1974; Feng et al., 1994). If we make the approximation that a particle set in a quadratic flow has a zero mean velocity relative to the fluid, due to the curvature of the velocity field, the fluid velocity will be (absolutely) higher on the wall side than on the centreline side in the reference frame of the particle (Fig. 5). By analogy with Saffman's force, this dissymmetry will cause a lower pressure on the side where the velocity of the fluid is higher, leading the particle to migrate away from the axis until the wall pushes it away.

This third type of lateral force on neutrally buoyant particles can be interpreted in terms of the stresslet velocity field induced by the rigid particle. This velocity field, equivalent to a force dipole on the particle, would yield no lateral motion in a simple shear, but causes the particle to migrate in the presence of a gradient of shear. The equilibrium position of a neutrally buoyant particle in Poiseuille flow will be a balance between the force due to the curvature directed towards the wall and the wall repulsion. When the particles are not neutrally buoyant, Saffman's force has to be added to the former lateral forces. The traditional pinch effect equilibrium position will consequently be moved towards the wall if the particle leads the flow and towards the axis if the particle lags the flow, in agreement with experimental results (Jeffrey and Pearson, 1965).

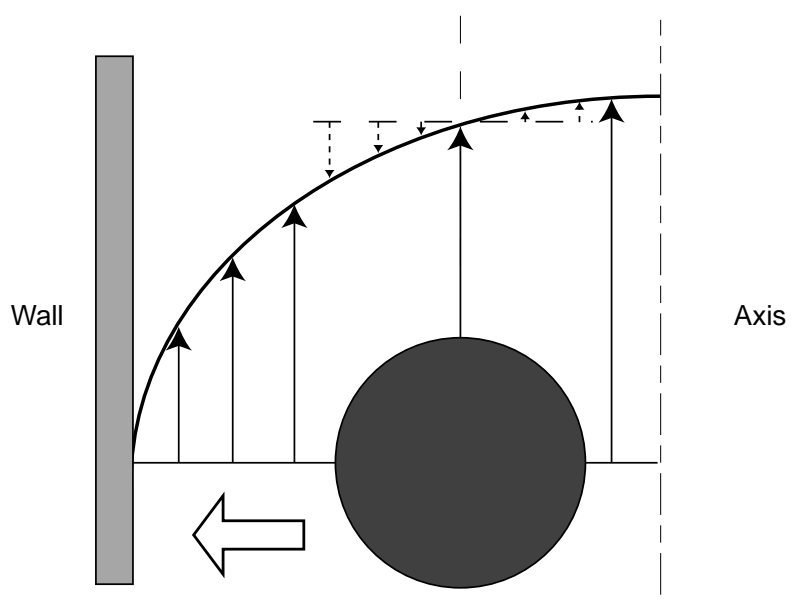

Figure 5

Force exerted on a neutrally buoyant rigid sphere in a quadratic flow: in the frame moving with the particle the fluid velocity is larger on the wall side due to the concavity of the velocity profile. 


\subsection{Influence of Inertia on Particle Migration}

All the studies mentioned above assumed finite but low Reynolds numbers. Under this assumption, the equilibrium position in pipe or channel flow can be found to be at $r=0.63 R$ from the centreline. Segré and Silberberg (1962) suggest that when the Reynolds number is increased the equilibrium position of a sphere in quadratic flow, is moved towards the wall, the effect beginning as soon as Re $>30$. Schonberg and Hinch (1989) and more recently Asmolov (1999) calculated the force on a neutrally buoyant particle in a two dimensional quadratic flow for Reynolds numbers up to 1500 by integrating the solution of the matched asymptotic expansion problem in Fourier space (the particle Reynolds number is assumed to be small). The calculations are made for channel flow, so a possible influence of curvature effects in the case of pipe flow cannot be captured. Their results show that the repulsive influence of the walls is strongly reduced for high Reynolds numbers, leading to equilibrium positions $x$ much closer to the wall (for instance $x=0.87 \mathrm{~L}$ for $\operatorname{Re}=500 . L$ is taken to be the half width of the channel with $L=R$ the radius of the pipe in the case of pipe flow). We present in Figure 6 the profiles of the force on a neutrally buoyant particle calculated for several Reynolds numbers following the method suggested by Schonberg and Hinch (1989) and Asmolov (1999). The calculations, based on a matched asymptotic expansion, assume a small particle Reynolds number: $\operatorname{Re}_{p}=\operatorname{Re}(a / L)^{2}<<1$, where $a$ is the particle radius. It can be shown that the force scales with $(d / D)^{-3}$, i.e. the particle size has a dramatic influence on the lateral force. The evolution of these profiles with the Reynolds number (Fig. 6) shows clearly that the equilibrium position is moved towards the wall (Asmolov, 1999). It can be noted that the lateral force scaled by $U^{2}$ decreases when the Reynolds number is increased: this indicates that though the lateral force is enhanced for large Reynolds numbers, it increases less quickly than $U^{2}$.

We have performed experimental measurements of concentration profiles in the case of neutrally buoyant particles in pipe flow for different Reynolds numbers. The particles were polystyrene spheres and were set in a mixture of $20 \%$ glycerol and $80 \%$ water matching their density. The particle volume fraction was of order $\varphi=5.10^{-3}$, so interparticle effects could be neglected. This very dilute suspension was flowed in a horizontal glass tube of inner diameter $D=8 \mathrm{~mm}$, and of length $L=2.6 \mathrm{~m}$. A laser was positioned at $2.5 \mathrm{~m}$ from the entrance, and produced a vertical sheet intersecting the pipe at a right angle. A plexiglas vessel was placed around the test section and filled with glycerol: the index of refraction of glycerol being close to that of glass, this allows a matching of the refraction at the cylindrical outer wall of the tube, and limits the deformation of the image. The cross section was filmed with a digital video camera (see Fig. 7), and the images were analyzed

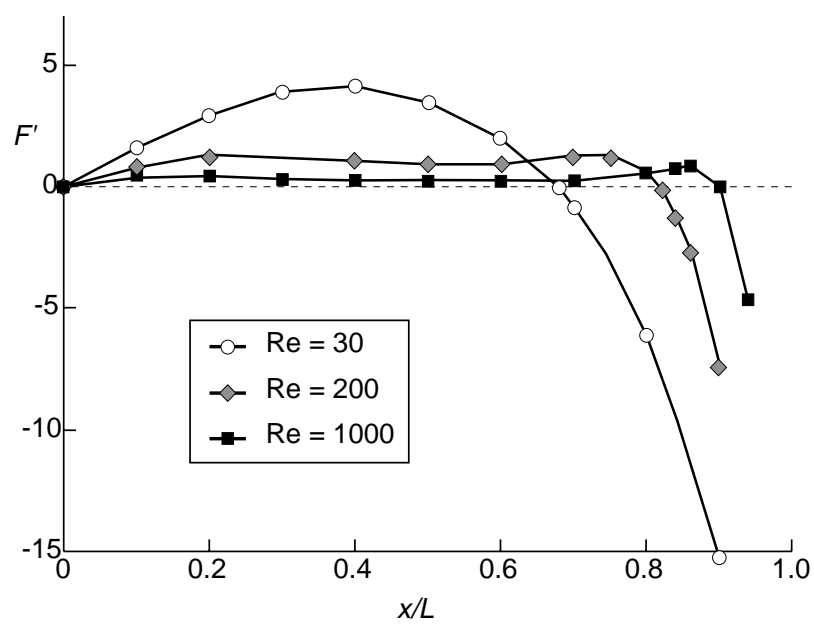

Figure 6

Non-dimensionalized force $F$ ' exerted on a neutrally buoyant particle in a quadratic flow as a function of position for different Reynolds numbers, where $F^{\prime}=(d / D)^{-4}\left(\rho U^{2} D^{2}\right)^{-1} F$, $d$ the particle diameter, $D$ the channel width, $\rho$ the fluid density and $U$ the maximum velocity of the quadratic flow. $x$ is the distance to the channel centre and $L=D / 2$ the half width of the channel. The equilibrium position is shifted closer to the wall when the Reynolds number is increased.

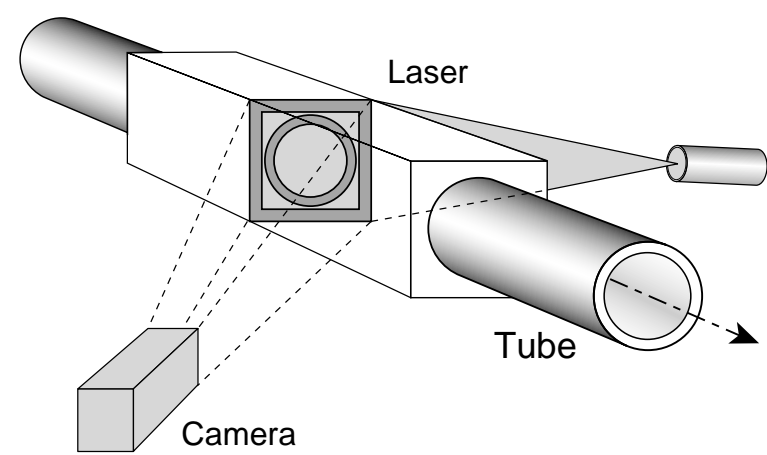

Figure 7

Measurement of particle position in the pipe: the camera records the position of the particles intersecting the vertical laser sheet.

with public domain software NIH Image to yield particle positions. Once calibrated to account for the deformation of the image due to refraction at the inner wall of the tube and the plane wall of the Plexiglas vessel, the data gives the cumulated particle distribution over the circular cross section. Typical distributions for two different Reynolds numbers are represented on Figure 8. 


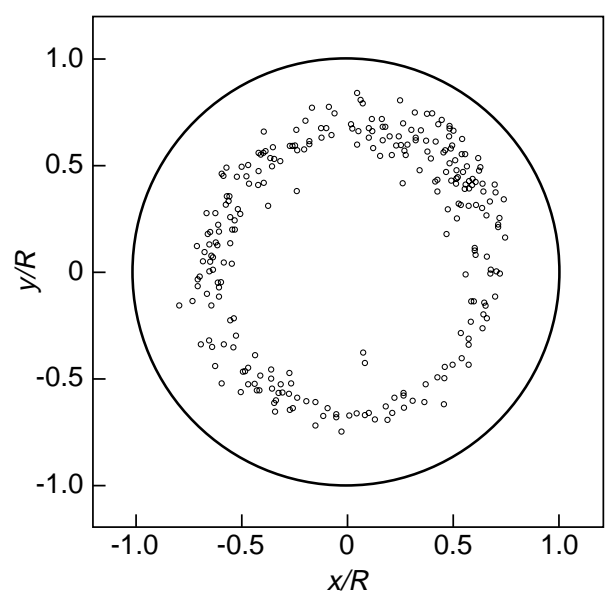

a)

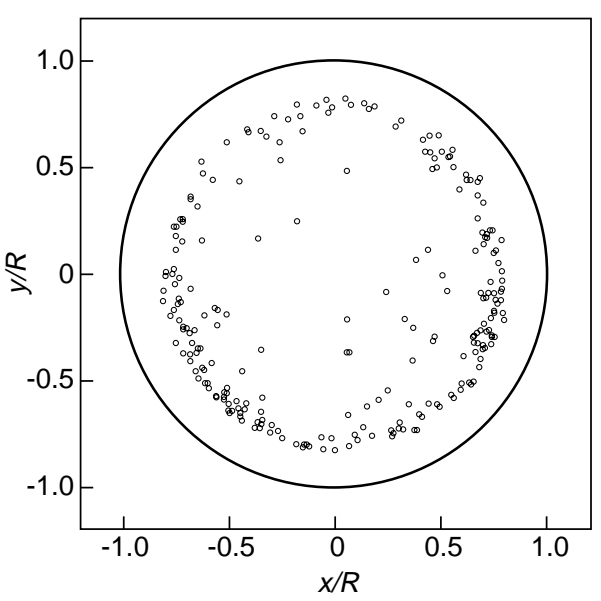

b)

Figure 8

Experimental distribution of particle centres (particle diameter is $900 \mu \mathrm{m}$ ) over a cross section of the flow observed for Reynolds numbers of a) $\operatorname{Re}=60$ and b) $\operatorname{Re}=350$. The lengths are scaled by the tube radius.

We compare these experimental results with the predictions of the theory by assuming that the influence of the curvature is small. These graphs are for particles of a diameter $d=$ $900 \mu \mathrm{m}$ in the pipe of diameter $D=8 \mathrm{~mm}$, i.e. rather large particles, for which migration is achieved more rapidly. Figure 8 shows clearly that there is a strong radial migration in our experimental conditions, and that for Reynolds numbers of 60 and 350, we obtain equilibrium positions $r_{e q}=0.64$ \pm 0.04 and $r_{e q}=0.78 \pm 0.04$ respectively. The value of $r_{e q}$ for $\operatorname{Re}=60$ is in agreement with the experimental data of Segré and Silberberg (1962), who measured a similar equilibrium position for this Reynolds number and with the same ratio of particle to pipe diameter. The comparison between $8 \mathrm{a}$ and $8 \mathrm{~b}$ shows that the increase in the Reynolds number shifts the equilibrium position towards the wall, as predicted by the theory. The prediction of the matched asymptotic expansion calculation for these Reynolds numbers is respectively of $r_{e q}=$ 0.71 and $r_{e q}=0.85$, in both cases larger than the measured experimental value. A possible explanation for this difference can be the relatively large size of the particles: the force calculations are performed in particular with the assumption of very small particle Reynolds number, which is not true for these particles for which $\operatorname{Re}_{p}=0.7$ and $\operatorname{Re}_{p}=4$ respectively. When the experiments are made with smaller particles (200 $\mu \mathrm{m})$, we observed that they yield equilibrium positions closer to that predicted by the theory. The discrepancies can also be due to the influence of the curvature of the pipe, neglected in the theory.

When the flow rate is further increased, we observed that particles were distributed not only on the previous equilibrium position but also on a second, smaller annulus (Fig. 9a). For a Reynolds number of 1500, this inner annulus is the only equilibrium position observed (Fig. 9b). A possible explanation for this new equilibrium position can be found on the force profiles of Figure 6. It can be seen that for a Reynolds number $\operatorname{Re}=1000$, there is a change in the concavity of the curve, leading to a region around $r=0.5 \pm 0.2 R$ where the radial force is much smaller than at the centre of the pipe. This region of low force can be observed from $\mathrm{Re}=500$. An explanation of the results of Figure 9 could then be that though particles still tend to leave the centre of the pipe, now they accumulate in this region of low radial force situated at $r=0.5 \pm 0.2 R$. However, we cannot exclude the possibility that this inner annulus be a real equilibrium position not captured by the 2D theory, and this issue remains an open question. The fact that the outer equilibrium position is not observed on Figure $9 \mathrm{~b}$ could be due to the strong velocity fluctuations of the particles at these Reynolds number, for which the system is close to the laminar-turbulent transition (Matas et al., 2003).

We have seen in this paragraph how inertially induced nonlinearity could generate lateral motion of a single particle. We now turn to the problem of lateral motion in nonNewtonian suspending liquids, where the nonlinearity arises from the rheology.

\subsection{Single-Particle Migration in Polymeric and Viscoelastic Fluids}

In applications where maintaining particles suspended for extended distances is an issue, with a number of examples in petroleum production including sand and other proppant transport in hydraulic fracturing, the suspending fluid is often a polymer solution or other strongly non-Newtonian liquid (for example oils gelled using surfactants). In shear-thinning or viscoelastic fluids, it has long been known that particles 


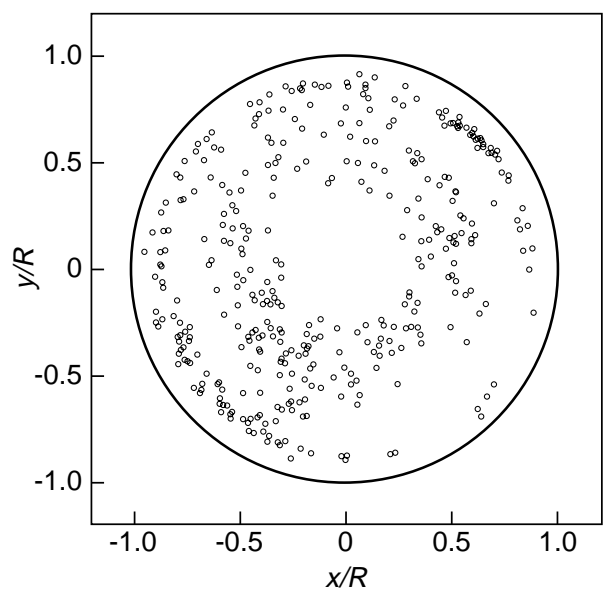

a)

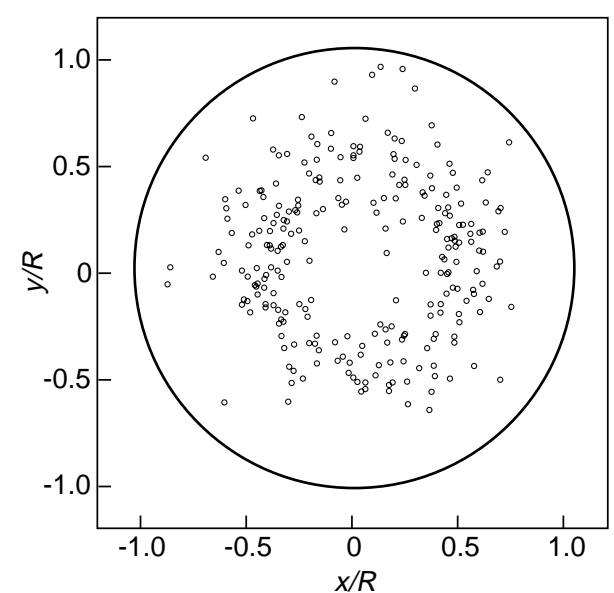

b)

Figure 9

Experimental distribution of particle centres (particle diameter is $550 \mu \mathrm{m}$ ) over a cross section of the flow for Reynolds numbers of a) $\operatorname{Re}=760$, and b) $\operatorname{Re}=1500$. For $\operatorname{Re}=760$, a second equilibrium position appears in the pipe at $r=0.5 \pm 0.2 R$. For $\operatorname{Re}=1500$ it is the only equilibrium position observed.

migrate laterally in pressure-driven flows. Here we touch briefly on these observations and efforts at their description.

Experimental work in this area by Mason and coworkers (e.g. Karnis and Mason, 1966) is among the best known. Gauthier, Goldsmith and Mason (1971) showed that for rigid spheres suspended in a predominantly shear-thinning fluid, migration is towards the wall of pressure-driven tube flow, while for a highly elastic liquid the migration is towards the tube centreline.

The experiments of Tehrani (1996) sought to examine the issue of elasticity upon cross-stream migration by varying the level of elasticity in aqueous solutions of guar (a naturally occurring polysaccharide) through variation of the crosslinking density induced by addition of borax (sodium borate). Tehrani found that for suspensions from $5-12 \%$ solid fraction, migration was towards the channel centreline for the moderately cross-linked fluids, while there was very little migration for very highly cross-linked and elasticallydominated fluids. It was argued that the migration requires both normal stress and a radial variation in the shear rate, with the apparent lack of migration in highly elastic fluids resulting from plug flow over a substantial portion of the interior of the pipe. Experiments on migration of solids suspended in viscoelastic media in other geometries include torsional flow studies of single particles (Karis, Prieve and Rosen, 1984) and the recent study by Kim (2001) showing strong outward radial migration of particles in suspensions of 10-30\% overall solid fraction subject to parallel-plate flow.

While many experiments were at volume fractions of order $10 \%$ or greater, migration behavior for an elastic fluid was shown in theoretical work by Ho and Leal (1976) to be describable at the single particle level using a second-order fluid constitutive model; this work indicated that it was a normal stress imbalance across the particle which results in the migration in viscoelastic fluid. Brunn (1980) has shown that the inclusion of interaction effects between particles or of a particle with the boundary leads to discrepancies between observation and theory. There is reason to expect progress in this area through a combination of techniques as numerical work has progressed to the point that Huang and Joseph (2000) have computed migration in nondilute suspensions in two-dimensional Poiseuille flow at finite Reynolds number and finite elasticity.

\section{COLLECTIVE EFFECTS AND MIGRATION}

\subsection{The Rheology of Suspensions}

When a rigid spherical particle is set in a shear flow, it does not possess any mechanism to relieve the local straining motion. Even a force free and torque free rigid particle in a sheared flow will create a disturbance in the velocity field which will eventually increase the dissipation of the system. In the limit of small concentrations, this added dissipation can be calculated by neglecting multi-particle effects. In 1906, Einstein showed that in this limit it was equivalent to an increase in the viscosity of the effective fluid: $\eta_{\text {eff }}(\varphi)=$ $\eta_{0}(1+5 / 2 \varphi)$, where $\varphi$ is the particle volume fraction.

The influence of particles on the rheology of a suspension for larger concentrations was quantitatively modelled by Krieger (1972), who proposed the following formula to take into account the added dissipation induced by spherical particles in a homogeneous suspension:

$$
\eta_{\text {eff }}(\varphi)=\eta_{0}\left(1-\varphi / \varphi_{m}\right)^{-1.82}
$$


where $\eta_{0}$ is the viscosity of the pure fluid, and $\varphi_{m}$ the maximum (close-packing) volume fraction, for which the viscosity of the system diverges, evaluated at $\varphi_{m}=0.68$ for hard spheres. This formula yields in the limit of low volume fractions $\eta_{\text {eff }}(\varphi) \approx \eta_{0}\left(1+\varphi / \varphi_{m}{ }^{* 1.82}\right) \approx \eta_{0}(1+2.68 \varphi)$, close to Einstein's formula $\eta_{\text {eff }}(\varphi)=\eta_{0}(1+2.5 \varphi)$. A number of other empirical formulae for the viscosity are typically similar to the Krieger result in taking a divergence at maximum packing and often also have similar forms of the divergence.

While studying the rheology of suspensions of neutrally buoyant hard spheres in the viscous regime, Gadala-Maria and Acrivos (1980) noticed that for volume fractions larger than $40 \%$, the viscosity (measured with a Couette dynamic viscometer) was decreasing with time under constant shear rate. This behavior was explained a few years later, when Leighton and Acrivos (1987) presented a model for shearinduced migration. They pointed out that in sheared concentrated suspensions, non-Brownian particles migrated from regions of high shear and high concentration to regions of low shear and low concentration. In the experiment of Gadala-Maria and Acrivos, this phenomenon caused a migration of the particles out of the sheared Couette gap, thus causing a decrease of the concentration of the sample, and hence a decreasing effective viscosity. This work was followed by a number of theoretical or numerical studies (Phillips et al., 1992; Phan-Tien and Zang, 1994; Nott and Brady, 1994; Mills and Snabre, 1995; Morris and Brady, 1998; Morris and Boulay, 1999) but also experimental works (Abbott et al., 1991; Altobelli et al., 1991; Koh et al., 1994; Hampton et al., 1997; Lyon and Leal, 1998 or Butler et al., 1999).

The first experimental studies to investigate the concentration profile in the flow of concentrated suspensions were based upon laser doppler anemometry measurements (Koh et al., 1994, Lyon and Leal, 1998). These studies observed a strong migration of the particles towards the centre of the pipe in Stokes flow, provided that the bulk concentration is large enough. Koh et al., (1994) for instance measured a volume fraction $\varphi$ of $60 \%$ at the centre of the pipe for a global volume fraction of $30 \%$, the largest reached in their work. The main experimental difficulties in this kind of study are the necessity to match the index of refraction of the fluid to that of the particles, and to ensure that the entry length necessary to reach a stationary concentration profile is smaller than the test section. Lyon and Leal (1998) managed to reach volume fractions of $50 \%$, for which they observed a considerably flattened velocity profile and concentrations close to close-packing at the centre of the channel (Fig. 10).

A number of studies avoided the problem of index matching by using nuclear magnetic resonance imaging (MRI) (Abbott et al., 1991; Sinton and Chow, 1991; Averbakh et al., 1997; Hampton et al., 1997; Butler et al., 1999). These studies confirm and complete the results obtained by LDA.
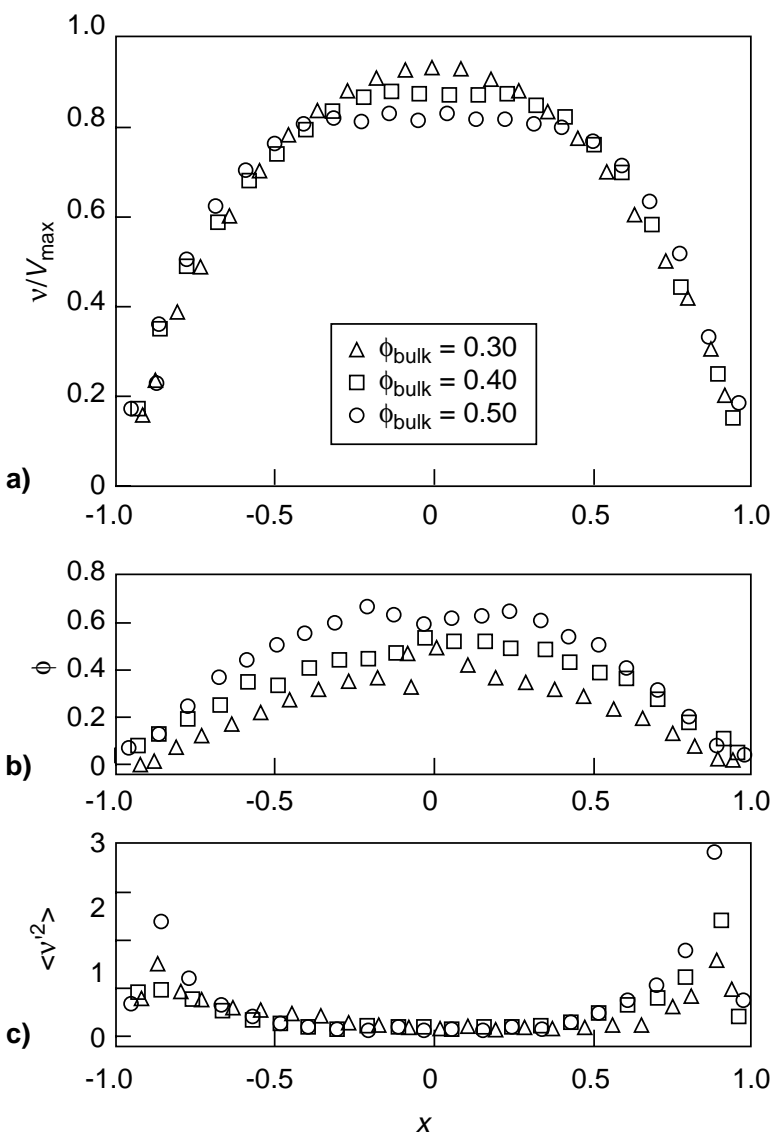

Figure 10

Experimental measurements of the velocity, the volume fraction and the velocity fluctuations for the channel flow of concentrated suspensions (from Lyon and Leal, 1998). For very concentrated suspensions the velocity profile is blunted. There is a strong migration of particles towards the centre of the channel.

NMR was also used by Han et al. (1999) who studied shear-induced migration in a broad range of concentration but also of Reynolds number. They show that for relatively low concentrations ( $\varphi$ about 10\%) there can be a competition between migration towards the centre of the pipe and the inertial migration towards $r=0.6 R$ (Segré and Silberberg's tubular pinch effect). For a given concentration, the balance between both effects is controlled by the particle Reynolds number, the tubular pinch being enhanced for inertial flows (Fig. 11).

\subsection{Mechanisms of Low Reynolds Number Migration}

Leighton and Acrivos (1987) proposed equations to describe the migration of particles in conditions of low Reynolds number. To account for the diffusion like behavior of the 

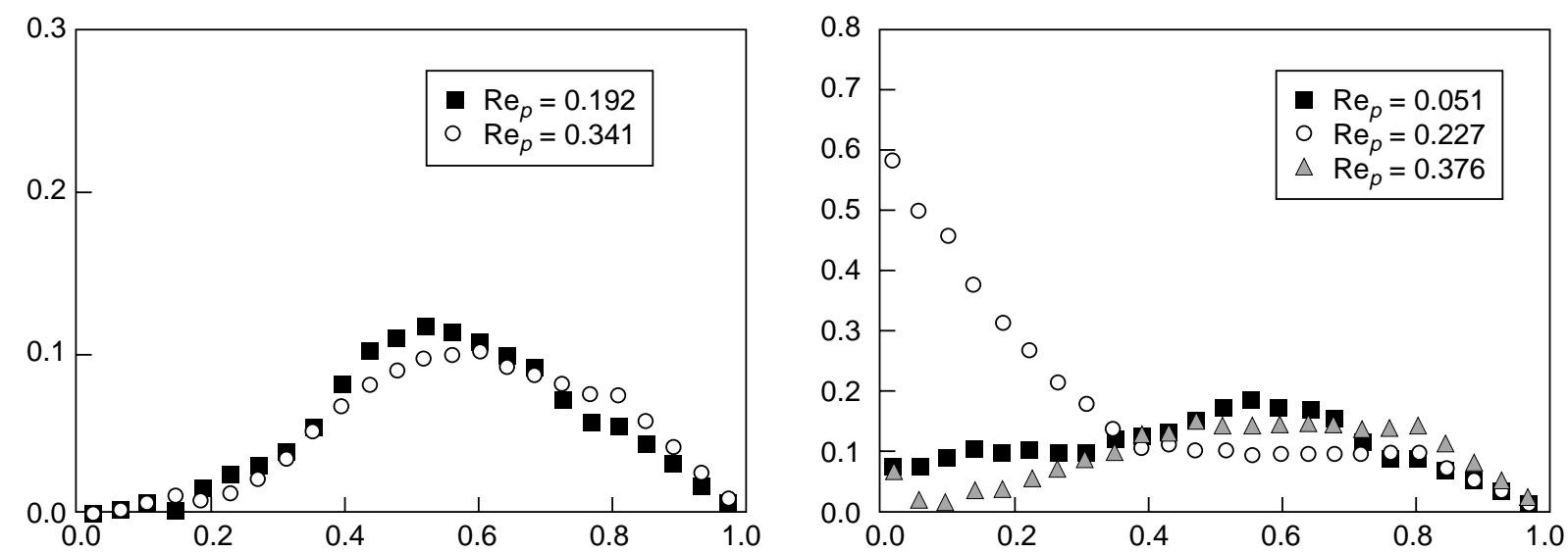

a)

b)

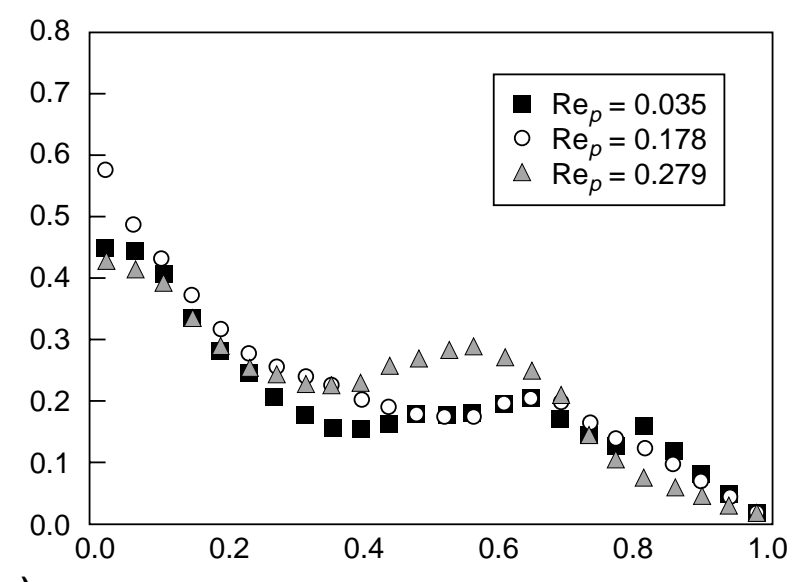

c)

Figure 11

Concentration profiles for the pipe flow of suspensions with average particle volume fraction a) $\varphi_{0}=0.06$, b) $\varphi_{0}=0.20$, and c) $\varphi_{0}=0.28$. Here the particle Reynolds number is defined as $\operatorname{Re}_{p}=\operatorname{Re}(d / D)$. For low particle volume fractions the pinch effect is the only migration observed. For particle volume fractions larger than $20 \%$ migration towards the centre of the pipe can occur if the flow is viscous enough. For larger concentrations there is a competition between both types of migrations (Han et al., 1999).

non-Brownian suspension in the experiment of Gadala-Maria and Acrivos (1980), they suggested that irreversible interparticle interactions due to surface roughness could lead to particle drift. Another possible source of irreversibility apart from particle roughness is three-body interactions (Phillips et al., 1992): whereas two-body interactions are known to be reversible in Stokes flow (for smooth particles), the presence of a third particle on two-body interactions can break this reversibility. An expression for the diffusive flux is derived in terms of the gradients in concentration and shear rate (corresponding to a spatially varying particle interaction frequency). The gradient in concentration-dependent viscosity will also create a diffusive flux (Leighton and Acrivos, 1987). This model has later been extended by Phillips et al. (1992) who apply it to several flow configurations. Physically, a given particle in a unidirectional shear flow will have a larger number of collisions on the side where the shear is larger, leading to migration towards the opposite side.

Nott and Brady (1994) developed a rather different approach (based on ideas in Jenkins and McTigue, 1990) which has been termed the suspension balance model: they consider the dynamics of the suspension not through diffusion but via mass, momentum and energy balances for the particulate phase and the entire suspension. In both Nott and Brady (1994) and Morris and Brady (1998), this model was used to predict the cross-stream migration of particles, with the latter study considering the balance of shear-induced migration with sedimentation of heavy particles. The basis for migration of solids at zero Reynolds number within this model is the presence of normal stresses due to the particles. 
Both of the noted studies employed a constitutive model which accounted for the normal stress through dependence upon the "suspension temperature," defined as the mean square velocity fluctuation (this quantity has been determined experimentally by Shapley et al., 2002). A more recent model (Morris and Boulay, 1999) accounts for the migration in more general flow conditions by showing that for flows with curved streamlines the normal stress differences are relevant. The constitutive modelling of the normal stresses is written directly in terms of the shear rate and concentration in this work, and the suspension temperature is thus not considered. The work of Zarraga et al. (2000) showed that the bulk of existing shear-induced migration results could be explained by considering bulk normal stresses, and thus support the modelling of migration as driven by particleinduced normal stresses.

\section{INFLUENCE OF SEDIMENTATION ON LATERAL MIGRATION}

Following the work of Segré and Silberberg (1962) on neutrally buoyant particles, a few authors investigated how sedimentation affected the migration phenomenon. The experimental studies of Jeffrey and Pearson (1965) and Aoki et al., (1979) on vertical pipe flow of a Newtonian fluid demonstrated that the equilibrium position of a rigid sphere could be observed to be closer to the axis or closer to the wall according to the experimental conditions. If the sphere is leading the flow the equilibrium is achieved closer to the wall, but if the sphere is lagging the flow equilibrium is observed to be closer to the channel axis. These results were obtained theoretically by Hogg (1994) for general flow configurations by a matched asymptotic expansion method. The results for nonneutrally buoyant particles differ from those for neutrally buoyant particles mainly due to the presence of a significant Saffman's lift in these situations. A much stronger lead or lag velocity than in the neutrally buoyant case can respectively displace the equilibrium position towards the wall or towards the channel centreline (Fig. 2). In the case where the pipe or channel is horizontal, lateral migration is controlled by gravity, the previously mentioned lateral forces being first order corrections (in the Reynolds number). The cylindrical symmetry in pipe flow is broken, and in a given section of the pipe there is a single equilibrium position, at the bottom of the pipe if particles are heavier than the fluid, to which all particles will eventually tend to migrate.

Results showing the interaction of sedimentation with shear-induced migration in the viscous regime have been obtained in both experimental and simulational studies. Leighton and Acrivos (1986) first described the viscous resuspension of particles at vanishing Reynolds number. Experiments using magnetic resonance imaging (MRI) by
Altobelli, Givler and Fukushima (1991) illustrated that a region of dense solids can be supported by a lessconcentrated (and therefore less-dense) layer owing to the interplay of sedimentation and shear-induced diffusion; Zhang and Acrivos (1994) modelled this phenomenon using the diffusive flux approach. This result was also seen in the Stokesian dynamics simulation of Morris and Brady (1998), where the phenomenon was modelled using the suspension balance approach. Viscous resuspension and particle migration in the context of a hydraulic fracture is considered in Miskin et al. (1996).

\section{REFERENCES}

Abbott, J.R., Tetlow, N,. Graham, A.L., Altobelli, S.A., Fukushima, E., Mondy, L.A. and Stephens, T S. (1991) J. Rheol., 35, 773.

Altobelli, S.A., Givler, R.C. and Fukushima, E., (1991) J. Rheol., 35, 721 .

Aoki, H., Kurosaki, Y. and Anzai, H. (1979) Bulletin of the JSME, 22 164, 206.

Asmolov, E.S. (1999) J. Fluid Mech., 381, 63-87.

Averbakh, A., Shauly, A., Nir, A. and Semiat, R. (1997) Int. J. Multiphase Flow, 23, 409-424.

Bingham, E.C., and Green, H. (1919) Proc. Amer. Soc. Test. Mater., 19, 640.

Bretherton, F.P. (1962) J. Fluid Mech., 14, 284.

Brunn, P. (1980) J. Non-Newtonian Fluid Mech, 7, 271.

Butler, J.E., Majors, P.D. and Bonnecaze, R.T. (1999) Phys. Fluids, 11, 2865.

Fåhraeus, R., and Lindqvist, T. (1931) Amer. J. Physiol., 96, 562.

Feng, J., Hu, H.H. and Joseph, D.D. (1994) J. Fluid Mech., 277, 271.

Gadala-Maria, F. and Acrivos, A., (1980) J. Rheol., 24, 799.

Gauthier, F., Goldsmith, H.L. and Mason, S.G. (1971) Trans Soc. Rheol., 15, 297.

Halow, J.S. and Wills, G.B. (1970) AIChE J., 16, 281.

Hampton, R.E., Mammoli, A.A., Graham, A.L., Tetlow, N. and Altobelli, S.A. (1997) J. Rheol., 41, 621.

Han, M., Kim, C., Kim, M. and Lee, S. (1999) J. Rheol., 43, 1157.

Ho, B.P. and Leal, L.G. (1974) J. Fluid Mech., 35, 365.

Ho, B.P. and Leal, L.G. (1976) J. Fluid Mech., 76, 783.

Hogg, A.J. (1994) J. Fluid Mech., 272, 285-318.

Huang, P.Y. and Joseph, D.J. (2000) J. Non-Newtonian Fluid Mech., 90, 159.

Jeffrey, R.C. and Pearson, J.R.A. (1965) J. Rheol., 22, 721.

Jenkins, J.T. and Mctigue, D.F. (1990) Two-Phase Flows and Waves, Springer, New York.

Karis, T.E., Prieve, D.C. and Rosen, S.L. (1984) J. Rheol., 28, 381.

Karnis, A., Goldsmith, H.L and Mason, S.G. (1966) Can. J. Chem. Eng., 44, 181.

Karnis, A. and Mason, S.G. (1966) Trans. Soc. Rheol., 10, 571.

Kim, S. and Karrila, S.J. (191) Microhydrodynamics: Principles and Selected Applications, Butterworth-Heinermann. 
Kim, C. (2001) Korea-Australia Rheol. J., 13, 19.

Koh, C.J., Hookham, P. and Leal, L.G. (194) J. Fluid Mech., 266, 1. Krieger, I.M. (1972) Adv. Colloid Interface Sci., 3, 111.

Leal, L.G. (1980) Ann. Rev. Fluid Mech., 12, 435.

Legendre, D. and Magnaudet, J. (1998) J. Fluid Mech., 368 , 81-126.

Leighton, D. and Acrivos, A. (1986) Chem. Eng. Sci., 41, 1377.

Leighton, D. and Acrivos, A. (1987) J. Fluid Mech., 181, 415-439.

Lyon, M.K. and Leal, L.G. (1998) J. Fluid Mech., 363, 25.

Matas, J.P. Morris, J.F. and Guazzelli, E. (2003) Phys. Rev. Lett., 90.

Maude, A.D. and Whitmore, R.L. (1956) Brit. J. Appl. Phys., 7, 98. Mills, P. and Snabre, P. (1995) J. Phys. II, 5, 1597.

Miskin, I., Elliott., L., Ingham, D.B. and Hammond, P.S. (1996) International Journal of Multiphase Flow, 22, 403-415.

Morris, J.F. and Brady, J.F. (1998) Int. J. Multiphase Flow, 24, 105 .

Morris, J.F. and Boulay, F. (1999) J. Rheol., 43, 1213.

Nott, P.R. and Brady, J.F. (1994) J. Fluid Mech., 275, 157.

Oliver, D.R. (1962) Nature, 194, 1269.

Phan-Tien, N., Fang, Z. (1994) J. Rheol., 40, 521.
Phillips, R.J., Armstrong, R.C., Brown, R.A., Graham, A.L. and Abbott, J.R. (1992) Phys. Fluids A, 4, 31.

Repetti, R.V. and Leonard, E.F. (1964) Nature, 203, 1346.

Rubinow, S.I. and Keller, J.B. (1961) J. Fluid Mech., 11, 447.

Saffman, P.G. (1965) J. Fluid Mech., 22, 385.

Schonberg, J.A. and Hinch, E.J. (1989) J. Fluid Mech., 203, 517.

Scott-Blair, G.W. (1930) J. Phys. Chem., 34, 1505.

Scott-Blair, G.W. (1958) Rheologica Acta, 1, 123-126.

Segré, G. and Silberberg, A. (1962) J. Fluid Mech., 14, 136.

Shapley, N.C., Armstrong, R.C. and Brown, R.A. (2002) J. Rheol., 46, 241.

Sinton, S.W. and Chow, A.W. (1991) J. Rheol., 35, 735.

Tachibana, M. (1973) Rheol. Acta, 12, 58-69.

Tehrani, M.A. (1996) J. Rheol., 40, 1057.

Vasseur, P. and Cox, R.G. (1976) J. Fluid Mech., 78, 385.

Zarraga, I.E., Hill, D.A. and Leighton, D.T. (2000) J Rheol., 44, 185.

Zhang, K. and Acrivos, A. (1994) Int. J. Multiphase Flow, 20, 579-591.

Final manuscript received in December 2003 